\title{
BMJ Open Cochlear implantation for tinnitus in adults with bilateral hearing loss: protocol of a randomised controlled trial
}

\author{
Kelly Assouly (1D , ${ }^{1,2,3}$ Adriana L Smit (D) , ${ }^{1,2}$ Inge Stegeman (D) ,,2 \\ Koenraad S Rhebergen, ${ }^{1,2}$ Bas van Dijk, ${ }^{3}$ Robert Stokroos (D) ${ }^{1,2}$
}

To cite: Assouly K, Smit AL, Stegeman I, et al. Cochlear implantation for tinnitus in adults with bilateral hearing loss: protocol of a randomised controlled trial. BMJ Open 2021;11:e043288. doi:10.1136/ bmjopen-2020-043288

- Prepublication history for this paper is available online. To view these files, please visit the journal online (http://dx.doi. org/10.1136/bmjopen-2020043288).

Received 03 August 2020 Revised 25 March 2021 Accepted 25 April 2021

A) Check for updates

C Author(s) (or their employer(s)) 2021. Re-use permitted under CC BY-NC. No commercial re-use. See rights and permissions. Published by BMJ.

\section{${ }^{1}$ Department of}

Otorhinolaryngology, Head and Neck Surgery, University Medical Center Utrecht, Utrecht, The Netherlands

${ }^{2}$ University Medical Center Utrecht Brain Center, University Medical Center Utrecht, Utrecht, The Netherlands

${ }^{3}$ Cochlear Technology Centre, Mechelen, Belgium

Correspondence to

Kelly Assouly;

k.k.s.assouly@umcutrecht.nl

\section{ABSTRACT}

Introduction Tinnitus is the perception of sound without an external stimulus, often experienced as a ringing or buzzing sound. Subjective tinnitus is assumed to origin from changes in neural activity caused by reduced or lack of auditory input, for instance due to hearing loss. Since auditory deprivation is thought to be one of the causes of tinnitus, increasing the auditory input by cochlear implantation might be a possible treatment. In studies assessing cochlear implantation for patients with hearing loss, tinnitus relief was seen as a secondary outcome. Therefore, we will assess the effect of cochlear implantation in patients with primarily tinnitus complaints. Method and analysis In this randomised controlled trial starting in January 2021 at the ENT department of the UMC Utrecht (the Netherlands), patients with a primary complaint of tinnitus will be included. Fifty patients (Tinnitus Functional Index (TFI) $\geq 32$, Beck's Depression Index $<19$, pure tone average at $0.5,1,2 \mathrm{kHz}$ : bilateral threshold between $\geq 40$ and $\leq 80 \mathrm{~dB}$ and hearing thresholds in the ear to be implanted $(\geq 4 \mathrm{kHz}) \geq 50 \mathrm{~dB}$ ) will be randomised towards cochlear implantation or no intervention. Primary outcome of the study is tinnitus burden as measured by the TFI. Outcomes of interest are tinnitus severity, hearing performances (tinnitus pitch and loudness, speech perception), quality of life, depression and patient-related changes. Outcomes will be evaluated prior to implantation and at 3 and 6 months after the surgery. The control group will receive questionnaires at 3 and 6 months after randomisation. We expect a significant difference between the cochlear implant recipients and the control group for tinnitus burden.

Ethics and dissemination This research protocol was approved by the Institutional Review Board of the University Medical Center (UMC) Utrecht (NL70319.041.19, V5.0, January 2021). The trial results will be made accessible to the public in a peer-review journal. Trial registration number Trial registration number NL8693; Pre-results.

\section{BACKGROUND}

Tinnitus is the perception of sound without an external stimulus, often experienced as a ringing or buzzing sound. ${ }^{12}$ It is a common symptom with an approximate prevalence of $10 \%-30 \%$, depending on the selected population, ${ }^{3}$ increasing to $30 \%$ of adults over the

\section{Strengths and limitations of this study}

$\Rightarrow$ The randomised controlled study allows for high quality assessment of outcomes of cochlear implantation for patients suffering primarily from tinnitus and secondarily from moderate to moderately severe bilateral hearing loss.

$\Rightarrow$ Outcomes of interest are not limited to tinnitus burden but also consider anxiety and depression, quality of life and patient-related changes.

$\Rightarrow$ The intervention can induce risks associated with surgery and a residual hearing deterioration in the ear implanted which will be monitored by electrocochleography measurement.

$\Rightarrow$ This study is a further step towards evidence-based medicine for the clinical efficacy of cochlear implants as a tinnitus treatment.

age of 50 years. ${ }^{4}$ Tinnitus can be chronic and disabling for those individuals affected by it. It is a complex condition, in which many components are responsible for perceived burden, like loudness, comorbidity and sleep problems. The heterogeneous aspect of the disease is also accountable for differences in the tinnitus itself: localisation, sound characteristics, temporal course and underlying cause. The tinnitus burden and the individual needs of patients for tinnitus-related healthcare are various. While the underlying aetiology of tinnitus is still debated, one hypothesis is that the tinnitus arises from changes in neural activity caused by reduced or lack of auditory input due to hearing loss which often accompanies the tinnitus. ${ }^{56}$ To date, the only evidence-based therapy for the reduction of tinnitus burden is cognitive behavioural therapy ${ }^{5-9}$ which is offered as standard clinical care in many countries in people with bothersome tinnitus. ${ }^{10}$ However, this therapy only improves tinnitus distress but does not reduce tinnitus loudness. ${ }^{11}$ Sound therapy is also considered as a recommendation for patients with hearing loss 
according to European guidelines but there is a lack of conclusive evidence. ${ }^{101213}$

Since auditory deprivation is thought to be one of the causes of tinnitus, increasing the auditory input by cochlear implantation might be a possible treatment option. This hypothesis is confirmed by observations in studies assessing the effectiveness of cochlear implantation to restore hearing function in case of bilateral deafness, where tinnitus reduction is one of the secondary outcomes. ${ }^{14}$ Analysing the effect of intracochlear electrical stimulation with a cochlear implant (CI) on primarily tinnitus complaints has been investigated by only few studies. All studies assessing the effect of cochlear implantation for tinnitus concerned cases with single-sided deafness ${ }^{15-20}$ or patients with asymmetrical hearing loss. ${ }^{6}$ They all reported a significant tinnitus reduction after implantation. So far, there is no high level of evidence of the effect of intracochlear stimulation as an intervention for primary tinnitus complaint in case of bilateral moderate to severe hearing loss. ${ }^{14}$

Above mentioned studies provide the first evidence of possible effectiveness of cochlear implantation for the reduction of tinnitus burden. To provide clear evidence of the effectiveness of cochlear implantation for the suppression of tinnitus complaints, a statistically powered study is needed aiming at patients with tinnitus as their primary complaint instead of hearing loss. To what extent electrical stimulation can reduce tinnitus in patients with bilateral moderate to severe hearing loss (just below the current CI indication), but with primary complaint of tinnitus, is unknown. ${ }^{21}$ Therefore, we aim to study the effect of cochlear implantation on tinnitus burden in patients suffering primarily from tinnitus and failed standard clinical care. For these patients which also have a bilateral moderate to severe hearing loss, a randomised controlled trial (RCT) will be conducted in which cochlear implantation will be compared with no intervention.

\section{METHOD AND ANALYSIS \\ Study objectives}

The primary objective of this study is to assess the effect of electrical stimulation by a CI on tinnitus burden, measured with the Tinnitus Functional Index (TFI) at 6 months after cochlear implantation. Secondary outcomes are to assess the effect of CI on tinnitus severity, tinnitus pitch and loudness, auditory function, speech recognition, quality of life, symptoms of depression and anxiety, patient reported change in order to attest treatmentrelated differences.

\section{Patient involvement}

Patients were not involved in the design, or conduct, or reporting or dissemination plans of the study.

\section{Study design and setting}

The study is a monocenter clinical trial performed in a tertiary referral clinic (university hospital) in the
Netherlands (University Medical Center Utrecht). The protocol is reported according to the Standard Protocol Items: Recommendations for Interventional Trials statement. ${ }^{22}$ In this RCT, patients will be randomised into groups: a CI group and a control group (figure 1). Twenty-five patients (CI group) shall receive a CI in the ear mostly affected by tinnitus. The other 25 patients (control group) shall follow a follow-up period of 6 months with no intervention. The follow-up sessions will take place 3 and 6 months after implantation to assess the primary outcome of tinnitus burden and secondary outcomes of quality of life, treatment-related outcomes and auditory function.

\section{Study population}

The study population consists of patients seeking help for tinnitus, presenting at the outpatient clinic of Ear, Nose and Throat (ENT) of the UMC Utrecht, The Netherlands. Fifty patients aged 18 or older with moderate to severe tinnitus and moderate to severe hearing loss will be included after fulfilling eligibility and informed consent. They must meet the following criteria to be eligible for the study at randomisation.

\section{Inclusion criteria}

The eligibility criteria for patients are:

- Patients aged 18 or older.

- Seeking help for tinnitus.

- Subjective tinnitus.

- Moderate to catastrophic tinnitus burden: TFI $>32$.

- Tinnitus duration $>1$ year and tinnitus stability $>6$ months.

- Hearing level (measured with a maximum of 3 months before eligibility assessment):

- Audiometry (pure tone average (PTA) at 0.5, 1, $2 \mathrm{kHz}$ ): bilateral threshold between $\geq 40$ and $\leq 80 \mathrm{~dB}$.

- Hearing thresholds in the ear to be implanted ( $\geq 4$ $\mathrm{kHz}) \geq 50 \mathrm{~dB}$.

- Hearing threshold stability (PTA $<5 \mathrm{~dB}$ change for 1 year in each ear).

- No to mild depression: Becks Depression Inventory (BDI) $<19$.

- Health status allows general anaesthesia and surgery for the cochlear implantation.

- Failure of regular tinnitus care (eg, psychological or sound therapy).

- Dutch language proficiency.

- Willingness and ability to participate in all scheduled procedures outlined in the protocol.

- Able to understand and sign informed consent.

\section{Exclusion criteria}

A potential patient who meets any of the following criteria will be excluded from participation in this study:

- Patient primary seeking help for non-tinnitus hearing problems.

- Abnormal cochlear anatomy (ie, ossification). 


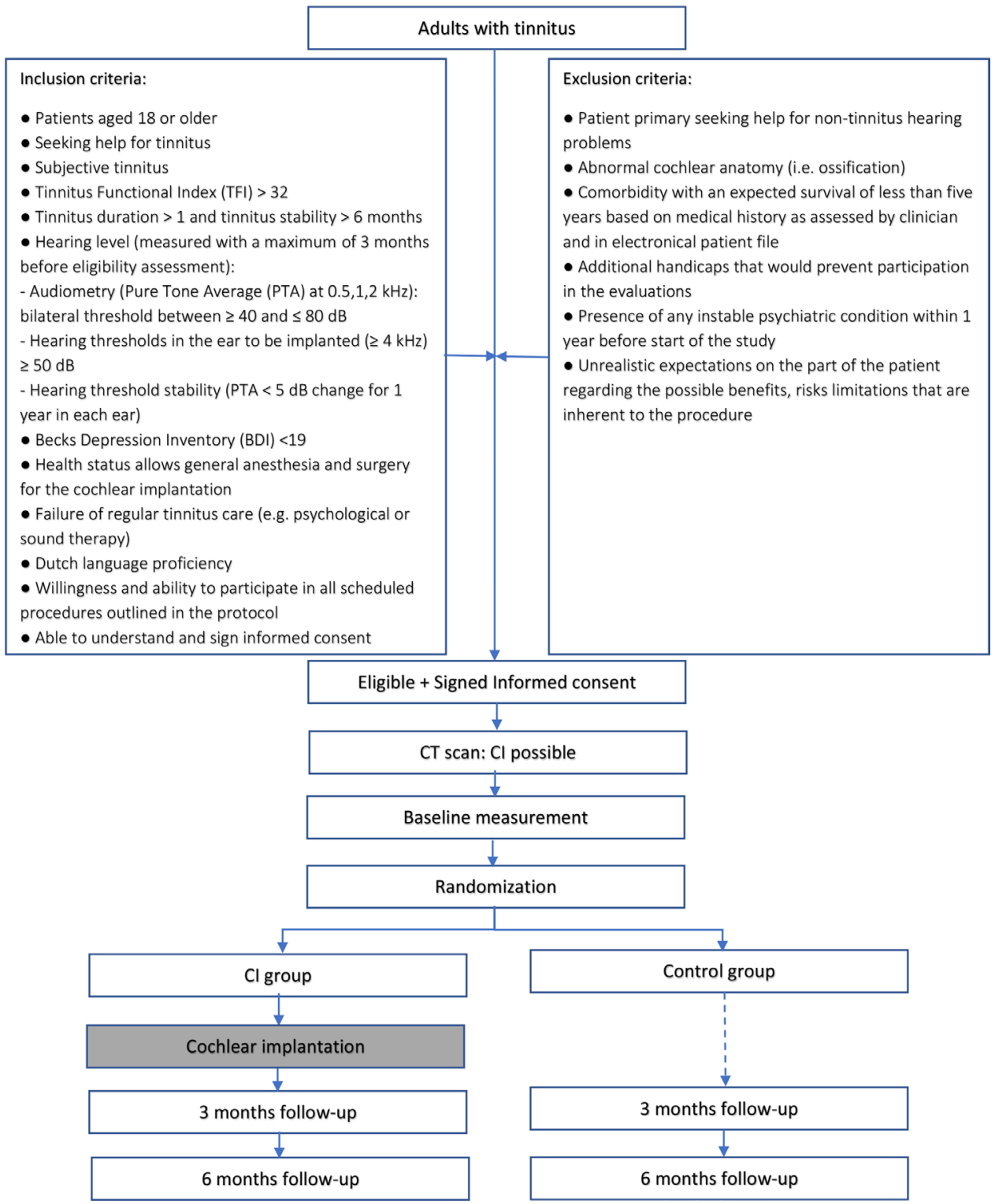

Figure 1 Study flowchart. Cl, cochlear implant group; control, control group.

- Comorbidity with an expected survival of less than 5 years based on medical history as assessed by clinician and in electronical patient file.

- Additional handicaps that would prevent participation in the evaluations.

- Presence of any unstable psychiatric condition within 1 year before start of the study.

- Unrealistic expectations on the part of the patient regarding the possible benefits, risks and limitations that are inherent to the procedure.

If a patient is eligible for the study, his/her otorhinolaryngologist will ask him/her to participate. The content of the study will be explained by the patient's otorhinolaryngologist who will provide him/her written patient information and the informed consent form. Patients will be given 2 weeks to consider participation. If a patient meets the criteria for inclusion and exclusion and wants to take part in the study, the patient will be asked to come to the UMC Utrecht for a CT scan to visualise the anatomy of the mastoid. If the patient's CT scan shows normal cochlear anatomy, he will, during the same visit, sign the informed consent with a member of the research team and receive a copy of the consent. After inclusion, baseline measurement will be performed where after randomisation will take place.

\section{Recruitment status and trial dates}

Patient enrolment started in January 2021 and will be completed in June 2022. The surveys and measurement will be performed until January 2023.

\section{Randomisation}

After inclusion and baseline measurement, patients will be randomly allocated into one of the two groups: CI group or control group. The randomisation will be computergenerated with block sizes of 4 and 6 and stratified for TFI score. A website randomisation programme, developed by Castor $\mathrm{EDC}^{23}$ will be used for randomisation. A study 
database was set up in Castor EDC to support allocation and concealment. Investigators enter information for each eligible patient and the randomisation assignment is revealed once the investigators validate the inclusion of the patient. The block design is unavailable to those who assign participants until the moment of assignment. Blinding is not possible during this study since both patients and caregivers will be able to see from outside whether patients have a CI or not.

\section{Intervention}

Patients allocated to the intervention group will receive a CI. The CI will be implanted on the most affected tinnitus side, and if equal tinnitus in the two ears, in the ear with the worst hearing loss. Hearing aid will be allowed in the contralateral ear. The cochlear implantation will be carried out under general anaesthesia after consent of the anesthesiologist and after determination of general health status. The standard surgical procedures for cochlear implantation will be followed. A retro-auricular incision will be made to expose the mastoid. The electrode will be inserted via a posterior tympanotomy and round window implantation by soft-surgery techniques. Intraoperatively, normal functioning of the device will be checked by measurement of impedance and neural response telemetry. Electrocochleography will also be recorded intraoperatively using Cochlear Research Platform (V.1.1). The CI used for the study consists of a Nucleus 7 sound processor and a CI622 implant with a slim straight electrode from Cochlear (or similar). Serial numbers of the CIs will be registered in the operating room report by the surgeon (standard clinical care for cochlear implantation) and in the master study file (product accountability). A postoperative cone beam CT of the mastoid will be planned to detail the electrode location within the cochlea.

One week after surgery patients from the intervention group will be checked at the outpatient department of the ENT to check for wound healing. The rehabilitation phase will start 4 weeks after surgery with a visit of the patient to the department of audiology to custom fit the processor software and then (bi)weekly till week 11 after surgery to fine tune the programming of the implant and improve speech perception. The CI fitting will not differ from the standard of care and will be optimised for every patient.

In the follow-up phase, the patients with CI will return to the UMC Utrecht 3 and 6 months after implantation to assess study outcome by the research team. The patients of the control group will come to the UMC Utrecht 3 and 6 months after randomisation to assess the same study outcome. A questionnaire will have to be filled in at home by the patients before every follow-up session at 3 and 6 months, as well as 2 weeks after surgery for the intervention group.

Participants are not allowed to start another tinnitus treatment during the study.

\section{Sample size}

To detect a clinically relevant difference of one grade (15 points) change measured with the TFI, ${ }^{24}$ in tinnitus burden at 6 months after cochlear implantation compared with the control group, with a power of $90 \%$ and alpha of $0.05,23$ patients are needed in both arms of the study. An acceptable SD was set at 15 , based on the results of a previous pilot study assessing CI for tinnitus patients. ${ }^{20}$ We will include 25 patients per arm, a 10\% margin, to include for possible lost to follow-up. Thereby, we expect patients to have a mean TFI at baseline of 50 points on TFI (grade 3 ) and a TFI decrease of 15 points at 6 months after intervention with a mean endpoint of 35 points on TFI (grade 2).

\section{Outcomes}

The following outcomes will be assessed at the baseline visit and follow-up visits at 3 and 6 months after randomisation (table 1). All measurements will be performed by the research team following the same protocol procedures.

\section{Primary outcome measure}

Our primary outcome is tinnitus burden as measured with the validated TFI. The TFI is a 25-item containing questionnaire with statements/questions about tinnitus burden. ${ }^{24}{ }^{25}$ The index is divided in eight subscale items: intrusive, sense of control, cognitive, sleep, auditory, relaxation and quality of life. Possible answers are ranging between 0 and 10, resulting in a maximum score of 100 , representing a maximum burden of tinnitus. This total score is then categorised into five different grades, indicating low to high burden.

\section{Secondary outcome measures Audiological tests}

Five audiological measurements are included in the study and are performed by an audiologist according to the ISO 16832:2006. ${ }^{26}$

\section{Pure tone audiometry}

The first evaluation is a pure tone audiometry at $0.25,0.5$, $1,1.5,2,4 \mathrm{kHz}$. This standard measurement evaluates the audible threshold of the patient by having patients indicating audibility for frequency-specific pure tone stimuli at different loudness level. The evaluation results in an audiogram which provides information about the hearing level of the patients.

\section{Speech recognition test in quiet and noise}

The second evaluation is a speech recognition test in quiet and noise. For the patients receiving a CI, postintervention assessments will be applied with the CI. The participant is listening at digits, Dutch words and sentences in a sound-treated booth. The loudness of the speech will change during the test in steps of $2 \mathrm{dBs}$, but the noise signal will be presented at a constant level of $65 \mathrm{~dB}$ Sound Pressure Level (SPL). The patient is asked to repeat back the words. The patient will perform the same test in two different conditions: with or without noise. This test results in a speech reception threshold obtained 
Table 1 Schedule of visits and assessments to measure study outcome per group

\begin{tabular}{|c|c|c|c|c|c|c|c|}
\hline & \multirow{2}{*}{$\begin{array}{l}\text { Baseline } \\
\text { Rx }\end{array}$} & \multicolumn{4}{|c|}{$\mathrm{Cl}$ group } & \multicolumn{2}{|l|}{ Control group } \\
\hline & & $\mathrm{Cl}$ & 2 weeks post $\mathrm{Cl}$ & 3 months post $\mathrm{Cl}$ & 6 months post $\mathrm{Cl}$ & Rx +3 months & Rx +6 months \\
\hline $\mathrm{Cl}$ (surgery) & & $x$ & & & & & \\
\hline Electrocochleography & & $x$ & & $x$ & $x$ & & \\
\hline Hearing level & & & & $x$ & $x$ & $x$ & $x$ \\
\hline Tinnitus loudness match & $x$ & & & $x$ & $x$ & $x$ & $x$ \\
\hline $\mathrm{TFI}^{*}$ & $x$ & & & $x$ & $x$ & $x$ & $x$ \\
\hline VAS tinnitus * & $x$ & & $x$ & $x$ & $x$ & $x$ & $x$ \\
\hline $\mathrm{SSQ}^{*}$ & $x$ & & & $x$ & $x$ & $x$ & $x$ \\
\hline $\mathrm{GBI}^{*}$ & & & & & $x$ & & \\
\hline $\mathrm{CGI}^{*}$ & & & & $x$ & $x$ & & \\
\hline ESIT-SQ* & $x$ & & & & & & \\
\hline
\end{tabular}

${ }^{*}$ Questionnaires $(\mathrm{Q})$ will be filled in at home.

BDI, Beck Depression Inventory; CGI, Clinical Global Impression; CI, cochlear implantation; e.o.s, end of study; EQ5D, Euro-Quality-of-life 5D; ESITSQ, ESIT Screening Questionnaire; GBI, Glasgow Benefit Inventory; HADS, Hospital Anxiety and Depression Scale; Rx, randomisation; SSQ, Speech, Spatial and Qualities Hearing Scale; TFI, Tinnitus Functional Index; VAS, Visual Analogue Scale.

by averaging the signal-to-noise ratio over the list of words presented in order to obtain a $50 \%$ correct score. The outcome will permit to set up a rehabilitation programme with a speech therapist for the intervention group.

\section{Electrocochleography}

Electrocochleography (ECochG) is a technique to record electrical potentials generated in the inner ear and auditory nerve in response to acoustic stimulation. ECochG measurement will be performed intraoperatively and at 3 and 6 months after cochlear implantation. The measure will be followed by conventional audiological examination. During the measurement postoperatively, the patient will be asked to sit comfortably on a chair and not move. The operator will install the earplug in the patient's ear and connect it to an audio cable attached to a sound processor. The sound processor will generate acoustic stimulation through the audio cable and the electrical responses will be recorded in real time via the Cochlear Research Platform (V.1.1, Cochlear ltd). The ECochG provides a measure of the cochlear function.

\section{Pitch match experiment}

Pitch match of tinnitus is performed to find the pitch corresponding to the tinnitus pitch of the patient. An acoustic pitch matching and an electric pitch matching will be performed in a sound-treated booth. The acoustic pitch matching will provide information about the frequency of the tinnitus perceived whereas the electric pitch matching will provide information about the pitch-matched electrode. The patient will be asked to concentrate on the predominant pitch of their tinnitus. Two tones will be presented at the same intensity level previously matched with tinnitus. The patient will indicate which option, the first or the second, sounds the closest in pitch by manipulating the response switch forward and backward. The difference between the first and the second will become smaller and smaller, until there is one frequency that matches best. Each stimulation will be performed two times (apical-to-basal and basal-to-apical to prevent octave-confusion). The pitch matched will be identified as the pitch resulting of the two runs. If the result of the two runs is not the same, the procedure will be repeated until finding a consistent result at least two times. $^{27}$

\section{Loudness match experiment}

Loudness match of tinnitus is performed to find the loudness corresponding to the tinnitus acoustically and electrically. ${ }^{28}$ The experiment uses the tinnitus pitch matched. The pure tones are initially presented at $6 \mathrm{~dB}$ above threshold. The patient is instructed to adjust the loudness of the comparison tones to match that of their tinnitus. The adjustment of the intensity is made in a range of $5 \mathrm{~dB}$ for rough determination and then $1 \mathrm{~dB}$ steps until a satisfactory loudness match in obtained.

\section{Cl usage}

The history of several user characteristics will be logged from the processor. This provides the following outcome parameters: 
- Time on air, providing the time the device was used in speech environment or the device was off.

- Scenes, providing the time spending in different environments: quiet, speech, noise, speech in noise, music and wind.

- Level of the environmental sound in dBA.

- Programme usage, providing a daily average on programme usage.

\section{Questionnaires}

Questionnaires will be sent by email to the study participants through the data management programme Castor EDC. ${ }^{23}$ If participants do not want to perform online questionnaires, they will receive paper versions of the questionnaires by postal services. All questionnaires will be in the Dutch language.

\section{Tinnitus questionnaire}

- The Visual Analogue Scale (VAS) tinnitus has two items. The patient answers two questions about tinnitus severity and intrusiveness using a visual analogue scale that ranges from 0 (not at all) to 10 (extremely).

\section{Tinnitus history}

- The ESIT Screening Questionnaire (ESIT-SQ) ${ }^{29}$ consists of 39 items relevant for tinnitus profiling including 17 general and 22 tinnitus-specific questions. Every question presents multiple choice. The test is used a baseline questionnaire and takes approximately $10 \mathrm{~min}$ to fill in.

\section{Patient reported benefits}

- The Clinical Global Impression (CGI) consists of a one-item observer-rated scale that measures global improvement or change (CGIC). ${ }^{30}$ The question is scored on a scale from 1 to 7,1 meaning 'very much improved' to 7 meaning 'very much worse'.

- The Glasgow Benefit Inventory (GBI) is a validated questionnaire reporting change in health-related quality of life postintervention. ${ }^{31}$ It consists of 18 questions scored on a 5-point Likert scale where 1 indicates 'much worse' and 5 is for 'much better'. The questionnaire presents three different items: general subscale, social support and physical health.

\section{Quality-of-life questionnaires}

- The Euro-Quality-of-life 5D (EQ5D) is a standardised measure of generic health status. It contains only five questions. Each question deals with a specific domain: mobility, self-care, usual activities, pain/discomfort and anxiety/depression. ${ }^{32}$ The patient must choose between different sentences which corresponds to his/her health condition. The last question is a self-report of the overall health status using a visual analogue scaling from 0 (the worst health you can imagine) to 100 (the best health you can imagine).

- The Speech, Spatial and Qualities Hearing Scale (SSQ) measures hearing-related quality of life and consists of three scales that assess different domains of hearing: (1) the speech hearing subscale consists of 15 questions that assess the ability to separate speech from competing noise in a wide range of listening contexts, (2) the spatial hearing subscale consists of 17 questions that assess the ability to locate sound sources and their direction of movement, (3) the quality of hearing subscale consists of 19 questions that assess naturalness and clarity of sound sources. ${ }^{33}$ Possible answers are scored using a visual analogue scale ranging from 0 (not at all) to 10 (excellent).

\section{Comorbid symptom scores}

- The BDI is a 21-item questionnaire used as an indicator of the severity of depression. ${ }^{34}$ Each question is scored on four points ranged between 0 (for example 'I do not feel sad') and 3 ('I am so sad') with a maximum of total score of 63 .

- The Hospital Anxiety and Depression Scale (HADS) is a 14-item screening tool for anxiety and depression symptoms in non-psychiatric clinical populations. ${ }^{35}{ }^{36}$ Each sentence is scored between 0 and 3 where 0 confirms the sentence and 3 disagrees with it.

\section{Statistical analysis}

Baseline characteristics per group will be described as means or medians, depending on the normality of the data and SD. Between-group mean differences will be calculated with $95 \%$ CIs. A p value $<0.05$ is considered statistically significant.

The primary outcome will be the difference in TFI score between the intervention at 6 months after cochlear implantation and the control group after 6 months of no intervention, a continuous variable. Differences between the control and intervention group will be calculated using the unpaired t-test and the Mann-Whitney $\mathrm{U}$ test. The secondary outcome measures will be the performances on the auditory tests and the questionnaires. Differences between groups will be calculated using the unpaired t-test and the Mann-Whitney $\mathrm{U}$ test. Withinsubject comparisons will entail differences of mean values. These will be analysed using paired t-tests for continuous measures.

Interim analyses on the safety data will be performed and reviewed by an external data safety monitoring board (DSMB). An interim analysis will be done every 6 months starting after the five first patients reached 6 months of follow-up. A statistician will perform non-parametric test on the aided speech perception of the implanted ear only, performed 6 months postimplantation to monitor functional hearing performance. The DSMB will advise on stopping the study if there is a risk for the patient's safety based on tinnitus worsening and deterioration of functional hearing.

Potential missing data will be handled using multiple imputation. Complete cases analyses will be done as a sensitivity analysis. All analyses will be performed on an intention-to-treat basis. 


\section{ETHICS AND DISSEMINATION}

\section{Protocol version}

The study will be conducted according to the principles of the Declaration of Helsinki (version 2013, Fortaleza) and in accordance with the Medical Research Involving Human Subjects Act (WMO). The research protocol was approved by the Institutional Review Board (IRB) of the UMC Utrecht (NL70319.041.19) and the Dutch competent authorities.

\section{Protocol amendment}

All amendments will be notified to the local Medical Research Ethics Committee (MREC). The data from this study will be used for publication in peer-reviewed international journals, preferably open-access. To diminish possible chance on publication bias, the study will be reported using the Consolidated Standards of Reporting Trials guidelines. ${ }^{37}$

\section{Confidentiality}

All data will be treated confidentially. The data will be encrypted by using an unique patient identification number. The analysis will be performed with these coded patient data. The key code will be safeguarded by the investigators. The paper data files and informed consents will be stored in a locked cabin in a locked room. The data will be stored on the investigator's computer as well, which is secured by a password and situated in a locked room. The handling of personal data will comply with the EU General Data Protection Regulation and the Dutch Act on Implementation of the General Data Protection Regulation, the Uitvoeringswet AVG, UAVG. The final trial dataset will be safeguarded and available to the principal investigator and approved members of the research team.

\section{Data monitoring and auditing}

The investigator will submit a summary of the progress of the trial to the accredited MREC once a year. Information will be provided on the date of inclusion of the first subject, numbers of subjects included and numbers of subjects that have completed the trial, serious adverse events (SAEs)/serious adverse reactions, other problems and amendments. Trial quality will be monitored independently by the Julius Clinical Centre (UMC Utrecht, the Netherlands) according to regulations by the UMC Utrecht and the Dutch government. The local monitor will check $50 \%$ of signed informedconsents, inclusion and exclusion criteria, source data and SAEs. Due to the highrisk nature of the study, an external DSMB will be in place to perform ongoing safety surveillance. An interim analysis will be performed by the statistician of the research group and will be analysed by the DSMB every 6 months after the fifth first inclusions.

\section{Adverse events}

Besides the normal risks associated with surgery and general anaesthesia, adverse events related to cochlear implantation will be monitored by assessment and documentation of intraoperative and postoperative complications and device failures. Deterioration of the hearing $<30 \mathrm{dBs}$ (PTA) is expected after implantation because of the cochlear trauma and should not be considered as an adverse event. ${ }^{38}{ }^{39}$ All adverse events will be followed until they have abated or until a stable situation has been reached. All cases of SAEs will be reported to the local IRB and the Dutch competent authorities.

\section{Trial status}

The study is currently in recruitment phase.

Contributors All authors (KA, ALS, IS, KSR, RS and BvD) developed the protocol. IS provided statistical expertise in clinical trial design. KA drafted the manuscript. All other authors revised themanuscript. All authors read and approved the final version.

Funding Part of cost involved of this study is funded by Cochlear Ltd. as a nonrestrictive research grant (IIR1975). Cochlear Ltd. did not and will-by a research contract-not have influence on the data collection, analysis, data interpretation and publication.

Competing interests KA received funding from the European Union's Horizon 2020 research and innovation programme under the Marie Sklodowska-Curie grant (agreement number 764604). KA and BvD are employed at Cochlear Technology Centre, Mechelen, Belgium.

Patient and public involvement Patients and/or the public were not involved in the design, or conduct, or reporting or dissemination plans of this research.

Patient consent for publication Not required.

Ethics approval This research protocol was approved by the Institutional Review Board (IRB) of the UMC Utrecht (NL70319.041.19, V5, January 2021).

Provenance and peer review Not commissioned; externally peer reviewed.

Open access This is an open access article distributed in accordance with the Creative Commons Attribution Non Commercial (CC BY-NC 4.0) license, which permits others to distribute, remix, adapt, build upon this work non-commercially, and license their derivative works on different terms, provided the original work is properly cited, appropriate credit is given, any changes made indicated, and the use is non-commercial. See: http://creativecommons.org/licenses/by-nc/4.0/.

\section{ORCID iDs}

Kelly Assouly http://orcid.org/0000-0003-2667-9499

Adriana L Smit http://orcid.org/0000-0001-9126-9969

Inge Stegeman http://orcid.org/0000-0001-5154-7178

Robert Stokroos http://orcid.org/0000-0001-8037-2573

\section{REFERENCES}

1 van den Berge MJC, Free $\mathrm{RH}$, Arnold R, et al. Cluster analysis to identify possible subgroups in tinnitus patients. Front Neurol 2017;8:1-7

2 Moller AR, Salvi R, De Ridder D, et al. Pathology of tinnitus and Hyperacusis-Clinical implications. Biomed Res Int 2015;2015:608437.

3 Møller AR. Epidemiology of Tinnitus in Adults BT - Textbook of Tinnitus. In: Møller AR, Langguth B, De Ridder D, et al, eds. New York, NY: Springer New York, 2011. : 29-37.

4 Davis AE. The epidemiology of tinnitus.. In: Tyler R, ed. Handb tinnitus. Singluar, 2000: 1-23.

5 Hoare DJ, Kowalkowski VL, Kang S, et al. Systematic review and meta-analyses of randomized controlled trials examining tinnitus management. Laryngoscope 2011;121:1555-64.

6 Mertens G, De Bodt M, Van de Heyning P. Cochlear implantation as a long-term treatment for ipsilateral incapacitating tinnitus in subjects with unilateral hearing loss up to 10 years. Hear Res 2016;331:1-6.

7 Dobie RA. A review of randomized clinical trials in tinnitus. Laryngoscope 1999;109:1202-11.

8 Hoare DJ, Edmondson-Jones M, Sereda M, et al. Amplification with hearing AIDS for patients with tinnitus and co-existing hearing loss. Cochrane Database Syst Rev 2014;50. 
9 Martinez-Devesa P, Perera R, Theodoulou M, et al. Cognitive behavioural therapy for tinnitus. Cochrane Database Syst Rev 2010:CD005233.

10 Cima RFF, Mazurek B, Haider H, et al. A multidisciplinary European guideline for tinnitus: diagnostics, assessment, and treatment. HNO 2019;67:10-42.

11 Fuller T, Cima R, Langguth B, et al. Cognitive behavioural therapy for tinnitus. Cochrane Database Syst Rev 2020;1:CD012614.

12 Sereda M, Xia J, El Refaie A, et al. Sound therapy (using amplification devices and/or sound generators) for tinnitus. Cochrane Database Syst Rev 2018;12:CD013094.

13 Hobson J, Chisholm E, El Refaie A. Sound therapy (masking) in the management of tinnitus in adults. Cochrane Database Syst Rev 2012;11:CD006371.

14 Ramakers GGJ, van Zon A, Stegeman I, et al. The effect of cochlear implantation on tinnitus in patients with bilateral hearing loss: a systematic review. Laryngoscope 2015;125:2584-92.

15 Ramos Macías A, Falcón-González JC, Manrique Rodríguez M, et al. One-Year results for patients with unilateral hearing loss and accompanying severe tinnitus and hyperacusis treated with a cochlear implant. Audiol Neurootol 2018;23:8-19.

16 Van de Heyning P, Vermeire K, Diebl M, et al. Incapacitating unilatera tinnitus in single-sided deafness treated by cochlear implantation. Ann Otol Rhinol Laryngol 2008;117:645-52.

17 Poncet-Wallet C, Mamelle E, Godey B, et al. Prospective multicentric follow-up study of cochlear implantation in adults with singlesided deafness: tinnitus and audiological outcomes. Otol Neurotol 2020;41:458-66.

18 Punte AK, De Ridder D, Van de Heyning P. On the necessity of full length electrical cochlear stimulation to suppress severe tinnitus in single-sided deafness. Hear Res 2013;295:24-9. doi:10.1016/j. heares.2012.08.003

19 Ahmed MFM, Khater A. Tinnitus suppression after cochlear implantation in patients with single-sided deafness. Egypt $J$ Otolaryngol 2017;33:61-6.

20 Arts RAGJ, George ELJ, Janssen M, et al. Tinnitus suppression by intracochlear electrical stimulation in single sided deafness - a prospective clinical trial: follow-up. PLoS One 2016;11:e0153131.

21 Sampaio ALL, Araújo MFS, Oliveira CACP. New criteria of indication and selection of patients to cochlear implant. Int $J$ Otolaryngol 2011;2011:1-13.

22 Chan A-W, Tetzlaff JM, Gøtzsche PC, et al. SPIRIT 2013 explanation and elaboration: guidance for protocols of clinical trials. BMJ 2013;346:e7586.

23 BV C. Castor electronic data capture. Amsterdam, The Netherlands, 2018.
24 Meikle MB, Henry JA, Griest SE, et al. The tinnitus functional index: development of a new clinical measure for chronic, intrusive tinnitus. Ear Hear 2012;33:153-76.

25 Rabau S, Wouters K, Van de Heyning P. Validation and translation of the Dutch tinnitus functional index. B-ENT [Internet]. , 2014: 10, 251-8. http://www.embase.com/search/results?subaction= viewrecord\&from $=$ export\&id $=$ L603263036

26 Valente D. International standard acoustics - loudness scaling by means 2006;2006.

27 Arts RAGJ, George ELJ, Chenault MN, et al. Optimizing intracochlear electrical stimulation to suppress tinnitus. Ear Hear 2015;36:125-35

28 Theelen-van den Hoek FL, Boymans M, Stainsby T, et al. Reliability of categorical loudness scaling in the electrical domain. Int $J$ Audiol 2014;53:409-17.

29 Genitsaridi E, Partyka M, Gallus S, et al. Standardised profiling for tinnitus research: the European school for interdisciplinary tinnitus research screening questionnaire (ESIT-SQ). Hear Res 2019;377:353-9.

30 Guy W. CGI clinical global impressions. ECDEU Assess Man 1976.

31 Hendry J, Chin A, Swan IRC, et al. The Glasgow benefit inventory: a systematic review of the use and value of an otorhinolaryngological generic patient-recorded outcome measure. Clin Otolaryngol 2016;41:259-75.

32 Health Policy. EuroQol - a new facility for the measurement of healthrelated quality of life 1990 .

33 Gatehouse S, Noble I. The speech, spatial and qualities of hearing scale (SSQ). Int J Audiol 2004

34 Beck AT, Steer RA, Ball R, et al. Comparison of Beck depression inventories -la and -II in psychiatric outpatients. J Pers Assess 1996;67:588-97.

35 Bjelland I, Dahl AA, Haug TT, et al. The validity of the hospital anxiety and depression scale. J Psychosom Res 2002;52:69-77.

36 Zigmond AS, Snaith RP. The hospital anxiety and depression scale. Acta Psychiatr Scand 1983;67:361-70.

37 Schulz KF, Altman DC, Moher D. Consort 2010 statement: updated guidelines for reporting parallel group randomised trials. Ital J Public Health 2010.

38 Ramos-Macías A, Borkoski-Barreiro SA, Falcón-González JC, et al. Hearing preservation with the slim modiolar electrode nucleus CI532 $₫$ cochlear implant: a preliminary experience. Audiol Neurooto 2017:22:317-25.

39 Jurawitz M-C, Büchner A, Harpel T, et al. Hearing preservation outcomes with different cochlear implant electrodes: Nucleus $₫$ Hybrid $^{\mathrm{TM}}$-L24 and nucleus Freedom ${ }^{\mathrm{TM}} \mathrm{Cl}$ 422. Audiol Neurotol 2014;19:293-309. 
Correction: Cochlear implantation for tinnitus in adults with bilateral hearing loss: protocol of a randomised controlled trial

Assouly K, Smit AL, Stegeman I, et al. Cochlear implantation for tinnitus in adults with bilateral hearing loss: protocol of a randomised controlled trial. BMJ Open 2021;11:e043288. doi: 10.1136/bmjopen-2020-043288

An amendment to the protocol resulted in the change of one inclusion criteria of the study. The inclusion criteria 'pure tone average at $0.5,1,2$ and $4 \mathrm{kHz}$ : bilateral threshold between 50 and $\leq 75 \mathrm{~dB}$ ' has been changed to 'pure tone average at $0.5,1,2 \mathrm{kHz}$ : bilateral threshold between $\geq 40$ and $\leq 80 \mathrm{~dB}$ and hearing thresholds in the ear to be implanted $(\geq 4 \mathrm{kHz}) \geq 50 \mathrm{~dB}$ '. The correction of the inclusion criteria does not change the aim and the design of the study, but for clarification, the following correction are noted:

1. The inclusion criteria in the 'Methods and analysis' section of the Abstract should be: (Tinnitus Functional Index (TFI) $>32$, Beck's Depression Index $<19$, pure tone average at $\mathbf{0 . 5 , 1 , 2} \mathbf{~ k H z}$ bilateral threshold between $\geq 40$ and $\leq \mathbf{8 0 ~} \mathrm{dB}$ and hearing thresholds in the ear to be implanted $(\geq 4 \mathrm{kHz}) \geq 50 \mathrm{~dB})$.

2. In the Method and analysis section, in the Inclusion criteria paragraph, the hearing level criteria should be:

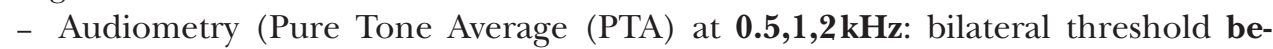
tween $\geq 40$ and $\leq 80 \mathrm{~dB}$.

- Hearing thresholds in the ear to be implanted $(\geq 4 \mathrm{kHz}) \geq 50 \mathrm{~dB}$

- Hearing threshold stability (PTA $<5$ dB change for 1 year in each ear).'

3. Figure 1 has been updated with the correction of the inclusion criteria.

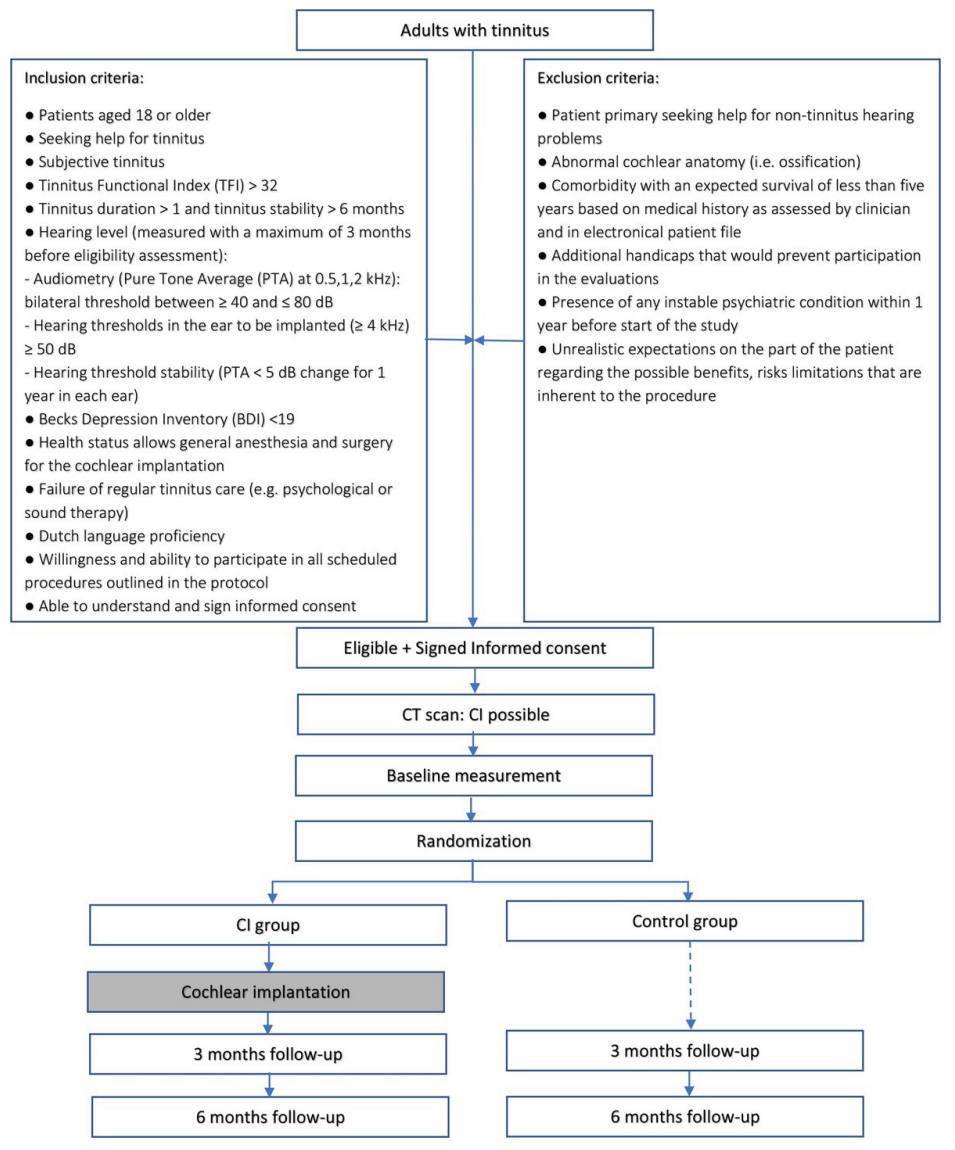


Open access This is an open access article distributed in accordance with the Creative Commons Attribution Non Commercial (CC BY-NC 4.0) license, which permits others to distribute, remix, adapt, build upon this work non-commercially, and license their derivative works on different terms, provided the original work is properly cited, appropriate credit is given, any changes made indicated, and the use is non-commercial. See: http://creativecommons.org/licenses/by-nc/4.0/.

(C) Author(s) (or their employer(s)) 2022. Re-use permitted under CC BY-NC. No commercial re-use. See rights and permissions. Published by BMJ.

BMJ Open 2022;12:e043288corr1. doi:10.1136/bmjopen-2020-043288corr1

(A) Check for updates 